\title{
Craniometria em cães (Canis familiaris). Aspectos em crânios mesaticéfalos
}

\section{Craniometric measurements in dogs (Canis familiaris). Features in the mesaticephalic skulls}

\author{
Bruno Cesar SCHIMMING ${ }^{1}$; José Ricardo de Carvalho PINTO E SILVA ${ }^{1}$ \\ ${ }^{1}$ Departamento de Anatomia do Instituto de Biociências de Botucatu, Botucatu, SP, Brasil
}

\begin{abstract}
Resumo
Neste estudo, foram utilizados 25 crânios de cães adultos, s.r.d., de ambos os sexos, todos mesaticéfalos. Foram realizadas medidas craniométricas para as diferentes partes do crânio. Os índices cefálicos e as proporções foram calculados. Obteve-se medidas a partir dos índices craniométricos ínio, bregma, násio, próstio, básio, eurio, zígio e pogônio. Calculou-se a altura do crânio (41,92 mm $\pm 3,9)$, o comprimento do crânio $(177,72 \mathrm{~mm} \pm 16,22)$, do neurocrânio $(96,84 \mathrm{~mm} \pm 7,25)$, do viscerocrânio $(79,24 \mathrm{~mm} \pm 8,72)$, basal (140,24 $\mathrm{mm} \pm 12,21)$, condilobasal $(149,32 \mathrm{~mm} \pm 12,21)$, do focinho $(69,84 \mathrm{~mm} \pm 7,07)$, dos ossos nasais $(50,08 \mathrm{~mm} \pm 6,77)$, da mandíbula $(129,88 \mathrm{~mm} \pm 16,3)$ e do palato $(76,84 \mathrm{~mm} \pm 7,01)$; e a largura do neurocrânio $(62,24 \mathrm{~mm} \pm 3,8)$ e zigomática $(95,44 \mathrm{~mm} \pm 7,85)$. Além disso, foram calculados os índices do crânio $(53,83 \mathrm{~mm} \pm 3,36)$, do neurocrânio $(64,48 \mathrm{~mm} \pm 4,69)$, facial $(121,06 \mathrm{~mm} \pm 9,18)$ e o basal $(44,64 \mathrm{~mm} \pm 4,17)$. Também foram realizadas medidas na área occipital, que incluíram a largura entre processos jugulares (41 mm $\pm 4,2)$, entre os côndilos occipitais $(35,48 \mathrm{~mm} \pm 3,21)$, e do forame magno $(17,48 \mathrm{~mm} \pm 2,27)$; a altura do triângulo occipital $(38,28 \mathrm{~mm} \pm 3,35)$, do forame magno (14,36 mm $\pm 1,07)$, o comprimento da incisura dorsal (16,1 $\mathrm{mm} \pm 1,12)$ e o índice do forame magno $(83,71 \mathrm{~mm} \pm 14,44)$. Embora com algumas diferenças nas mensurações, não houve diferença significativa entre as dimensões observadas e as relatadas para crânios mesaticéfalos.
\end{abstract}

Palavras-chave: Craniometria. Cães. Crânio mesaticéfalo.

\begin{abstract}
In this study, a total of 25 skulls of the adult, mongrel and mesaticephalics dogs were used. Craniometric measurements for different parts of the skull were made. Cephalic indices and ratios were calculated. Certain points and landmarks on the skull are recognized in making linear measurements and are inion, bregma, nasion, prosthion, pogonion, basion, euryon, and zygion. Skull height $(41,92 \mathrm{~mm} \pm 3,9)$, skull length $(177,72 \mathrm{~mm} \pm 16,22)$, neurocranium $(96,84 \mathrm{~mm} \pm 7,25)$, viscerocranial $(79,24 \mathrm{~mm} \pm 8,72)$, basal length $(140,24 \mathrm{~mm} \pm 12,21)$, condylobasal $(149,32 \mathrm{~mm} \pm 12,21)$, snout $(69,84$ $\mathrm{mm} \pm 7,07)$, nasals $(50,08 \mathrm{~mm} \pm 6,77)$, mandibular $(129,88 \mathrm{~mm} \pm 16,3)$, and of palatal $(76,84 \mathrm{~mm} \pm 7,01)$; width of neurocranium $(62,24 \mathrm{~mm} \pm 3,8)$, and zygomatic $(95,44 \mathrm{~mm} \pm 7,85)$ were obtained. Therefore, were calculated cranial index $(53,83 \mathrm{~mm} \pm 3,36)$, neurocranium $(64,48 \mathrm{~mm} \pm 4,69)$, facial $(121,06 \mathrm{~mm} \pm 9,18)$, basal $(44,64 \mathrm{~mm} \pm 4,17)$, width of the jugular processes $(41 \mathrm{~mm} \pm 4,2)$, of occipital condyles $(35,48 \mathrm{~mm} \pm 3,21)$, and of the foramen magnum (17,48 $\mathrm{mm} \pm 2$,27); height of the occipital triangle $(38,28 \mathrm{~mm} \pm 3,35)$, of the foramen magnum $(14,36 \mathrm{~mm} \pm 1,07)$; length of the dorsal notch $(16,1 \mathrm{~mm} \pm 1,12)$, and foramen magnum index $(83,71 \mathrm{~mm} \pm 14,44)$. Although with some differences in measurements, no significant difference was observed between the dimensions and reported to mesaticephalic skulls.
\end{abstract}

Keywords: Craniometric measurements. Dogs. Mesaticephalic skull.

\section{Introdução}

O crânio é a parte mais importante, complexa e especializada do esqueleto. Ele abriga o encéfalo e os órgãos sensoriais da audição, equilíbrio, visão, olfato e gustação, além de órgãos dos sistemas respiratório e digestório. É basicamente dividido em uma região facial e uma neural ${ }^{1}$.

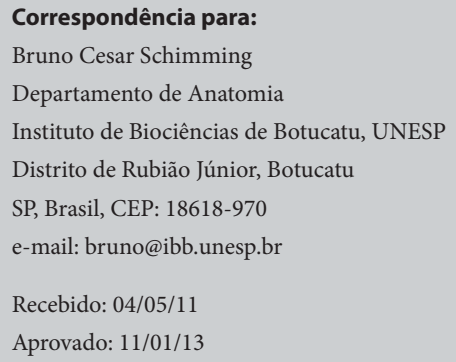


Os cães apresentam uma grande variedade de raças, o que reflete em uma diversidade de formas de crânios e de cabeças. Assim, a forma do crânio em cães varia consideravelmente de acordo com a raça e características individuais, muito mais do que em outras espécies de animais domésticos. O crânio do cão possui formato característico relacionado à raça. Ainda assim, existem diferenças individuais quanto ao tamanho e forma. A forma do crânio é o critério mais importante utilizado para se determinar o padrão racial em cães. Uma das principais afirmações na avaliação das características raciais de cães puros e mistos é a craniometria ${ }^{2}$. Com base em certos pontos craniométricos e marcos ósseos do crânio é possível estabelecer diversas medidas lineares. Estas medidas caracterizam as diferenças raciais no cão e relacionam-se com o comprimento relativo da parte facial, o que permite que se possa identificar raças dolicocefálicas, braquicefálicas e mesaticefálicas. A terminologia do formato do crânio em mesaticefálico, dolicocefálico e braquicefálico foi adotada pela Medicina Veterinária no final do século XIX ${ }^{3}$. As raças dolicocefálicas são as que possuem cabeça estreita e longa, por exemplo, o Collie. As raças mesaticefálicas, como o Beagle e o Setter, são aquelas que possuem cabeças com proporções médias, já as raças braquicefálicas, como Pequinês, possuem cabeça curta e larga ${ }^{1}$. Hofmann-Appollo ${ }^{4}$ afirma que o grupo dos cães mesaticefálicos tem formato craniano intermediário, comportando-se morfologicamente de maneira semelhante às diversas espécies de canídeos silvestres do gênero Canis. Estas variações fizeram com que o crânio do cão despertasse interesse em muitos pesquisadores $^{5,6,7,8,9}$.

A partir da caracterização do crânio do cão, citada anteriormente, objetivou-se com este estudo obter mensurações em crânios mesaticéfalos de cães sem raça definida, visando contribuir para a anatomia comparada, em especial para a anatomia canina, com uma possível padronização cranial de cães mesaticéfalos com ou sem raça definida.

\section{Material e Método}

Foram usados 25 crânios de cães adultos, sem raça definida, sem distinção de sexo, todos pertencentes ao grupo dos mesaticéfalos. Os crânios faziam parte do acervo ósseo do Laboratório de Anatomia Veterinária do Departamento de Anatomia, do Instituto de Biociências de Botucatu, UNESP. Realizou-se uma seleção prévia dos crânios com o objetivo de classificá-los como mesaticéfalos.

As medidas craniométricas para as diferentes partes do crânio foram realizadas com o auxílio de paquímetro digital (Digimess ${ }^{\circ}$ ) e segundo critérios estabelecidos por vários autores $1,6,7,8,9,10,11,12,13,14,15$. Os índices cefálicos foram calculados de acordo com fórmulas citadas em relatos anteriores ${ }^{1,6,8,9,10,15}$. Todas as características foram expressas em média e desvio-padrão. Os pontos de medidas craniométricos utilizados foram os mesmos citados em trabalhos prévios ${ }^{8,9,15}$.

\section{Definição dos pontos craniométricos usados para medidas do crânio (Figura 1)}

Ínio (I): ponto central na protuberância occipital externa;

Bregma (B): junção no plano mediano das suturas frontoparietais direita e esquerda;

Násio $(\mathrm{N})$ : junção no plano mediano das suturas frontonasais direita e esquerda;

Próstio (P): extremidade rostral da sutura interincisiva, entre as raízes dos dentes incisivos superiores centrais;

Básio (BA): meio da margem ventral do forame magno;

Eurio (E): ponto mais lateral do neurocrânio;

Zígio (Z): ponto mais lateral do arco zigomático;

Pogônio (PO): parte mais rostral da mandíbula, na sínfise, entre as raízes dos dentes incisivos inferiores centrais 


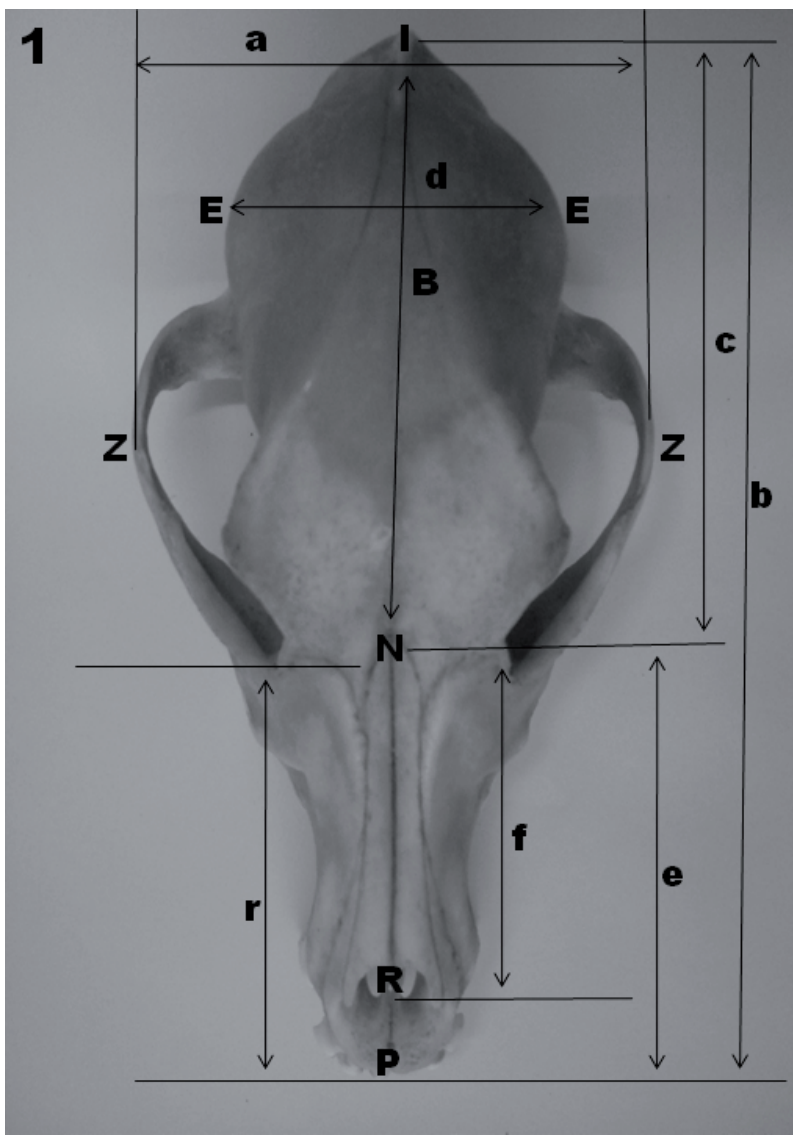

Figura 1 - Pontos antropométricos e mensurações em crânios mesaticéfalos de cães sem raça definida (vista dorsal). Observe I (ínio), E (eurio), Z (zígio), B (bregma), $\mathbf{N}$ (násio), $\mathbf{R}$ (rínio), $\mathbf{P}$ (próstio), a (largura máxima zigomática), b (comprimento do crânio), c (comprimento do neurocrânio), d (largura máxima do neurocrânio), e (comprimento do viscerocrânio), $\mathbf{f}$ (comprimento nasal) e $\mathbf{r}$ (comprimento do focinho)

\section{Medidas lineares utilizadas no crânio (Figuras 1, 2,} 3 e 4 )

As seguintes medidas foram obtidas:

altura do crânio: meio do meato acústico externo ao bregma

comprimento do crânio: ínio ao násio

comprimento do neurocrânio: básio ao násio comprimento do viscerocrânio: násio ao próstio largura do neurocrânio: eurio ao eurio largura zigomática: zígio ao zígio comprimento da base do crânio: básio ao próstio

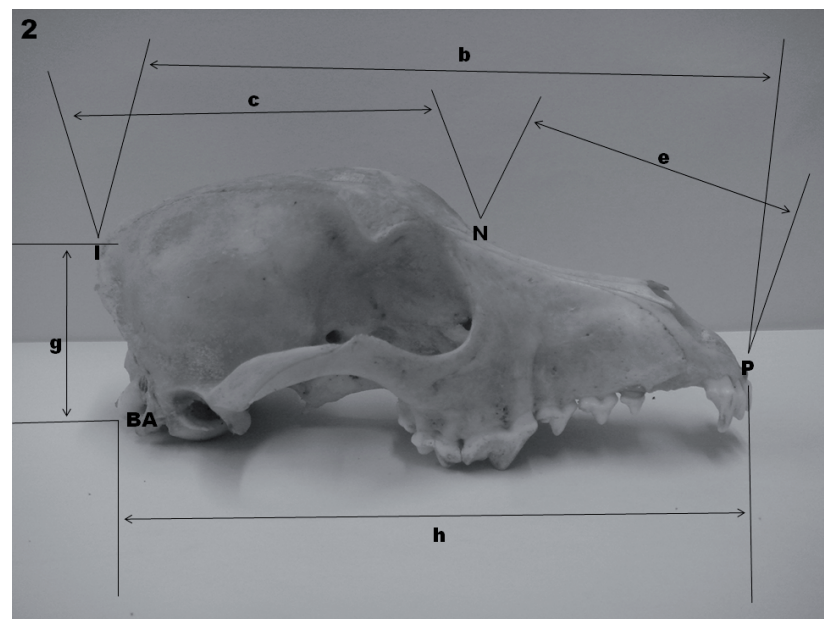

Figura 2 - Pontos antropométricos e mensurações em crânios mesaticéfalos de cães sem raça definida (vista lateral): I (ínio), $\mathbf{N}$ (násio), $\mathbf{P}$ (próstio), BA (básio), b (comprimento do crânio), c (comprimento do neurocrânio), e (comprimento do viscerocrânio), g (altura do crânio) e h (comprimento condilobasal)

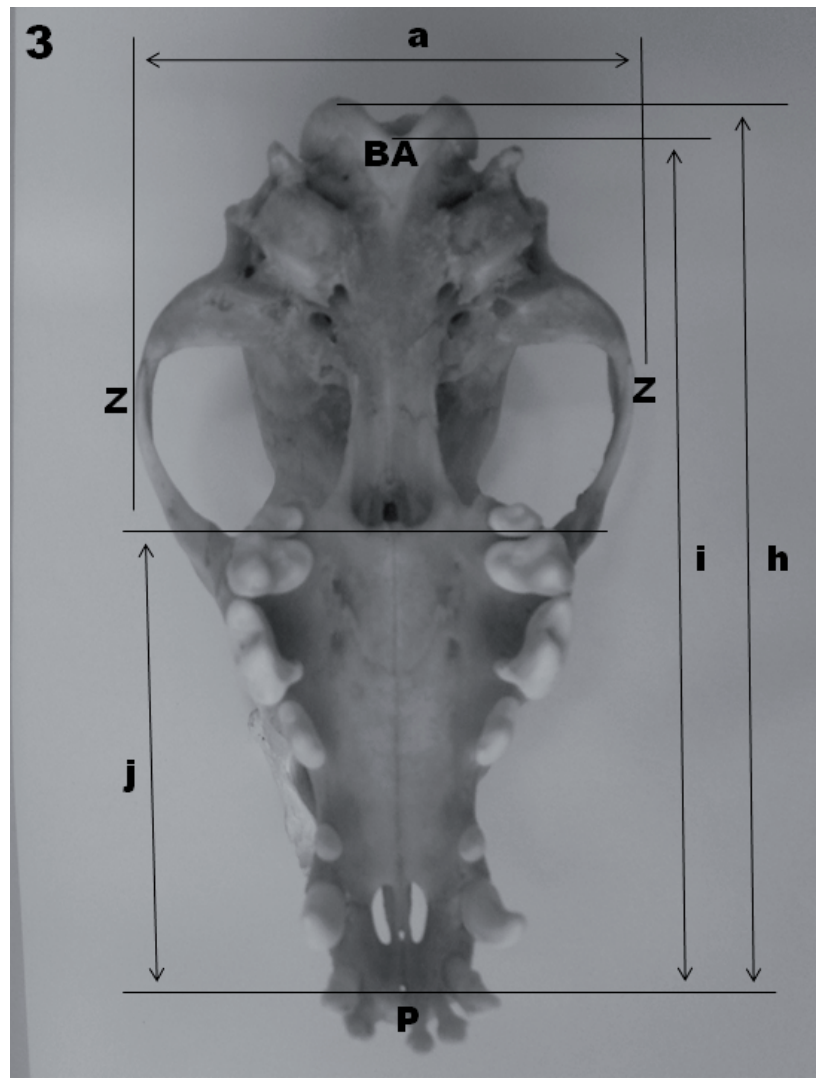

Figura 3 - Pontos antropométricos e mensurações em crânios mesaticéfalos de cães sem raça definida (vista ventral): BA (básio), Z (zígio), $\mathbf{P}$ (próstio), a (largura máxima zigomática), $\mathbf{h}$ (comprimento condilobasal), i (comprimento da base do crânio) e j (comprimento do palato) 


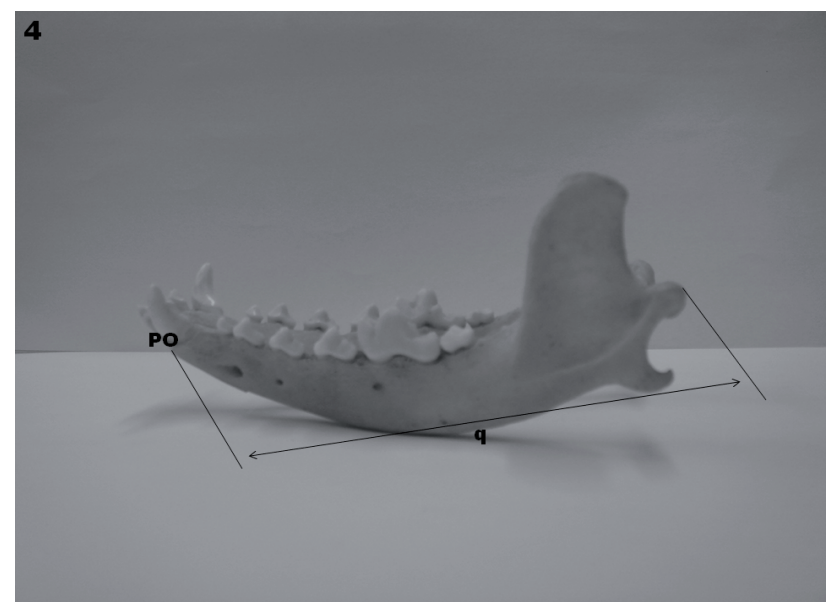

Figura 4 - Ponto antropométrico e mensurações em mandíbula de crânios mesaticéfalos de cães sem raça definida (vista lateral): PO (pogônio) e $\mathbf{q}$ (comprimento da mandíbula)

comprimento condilobasal: próstio às bordas caudais dos côndilos occipitais

comprimento do focinho: borda oral da órbita (mediana) ao próstio

comprimento dos ossos nasais: násio ao rínio

comprimento da mandíbula: margem caudal do côndilo ao pogônio

comprimento do palato: espinha nasal posterior do palatino ao próstio

\section{Medidas da área occipital (Figura 5)}

Também foram realizadas medidas na área occipital, que incluíram a largura entre processos jugulares, entre os côndilos occipitais e do forame magno; a altura do triângulo occipital e do forame magno e o comprimento da incisura dorsal ao forame magno.

\section{Índices calculados}

Além disso, foram calculados o índice do crânio (largura zigomática x 100/comprimento do crânio); do neurocrânio (largura do neurocrânio x 100/comprimento do neurocrânio); facial (largura zigomática x 100/comprimento do viscerocrânio); basal (largura do neurocrânio x 100/comprimento basal) e do forame magno (altura do forame magno x 100/maior largura do forame magno).

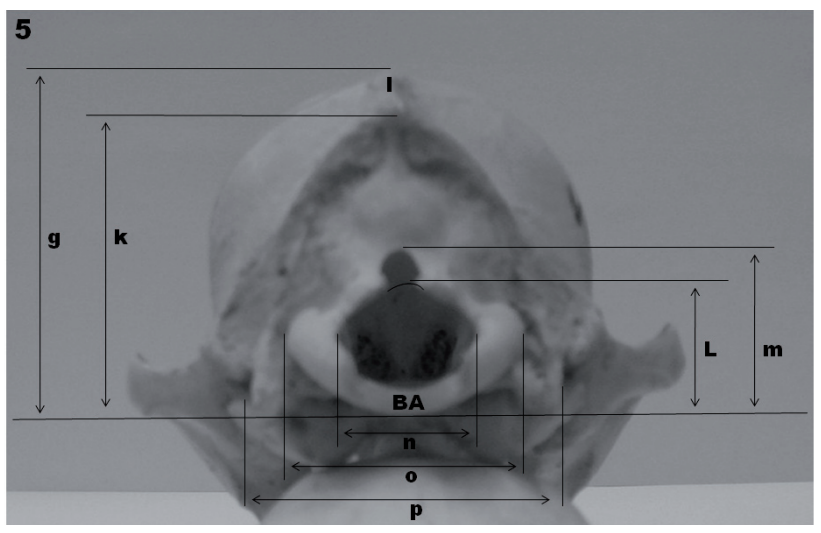

Figura 5 - Vista da área occipital em crânios mesaticéfalos de cães sem raça definida mostrando as mensurações craniométricas: BA (básio), I (ínio), g (altura do crânio), k (altura do triângulo occipital), 1 (altura do forame magno), m (comprimento da incisura dorsal), n (largura máxima do forame magno), o (largura máxima entre os côndilos occipitais) e p (largura máxima entre os processos jugulares)

\section{Resultados}

Observou-se nos crânios mesaticéfalos estudados comprimento médio de $177,72 \mathrm{~mm} \pm 16,22$ e largura zigomática de $95,44 \mathrm{~mm} \pm 7,85$. As mensurações craniométricas foram avaliadas e apresentadas como média \pm desvio-padrão (Tabela 1 ).

Os índices craniais foram calculados, obtendo-se índice cranial de 53,83 $\mathrm{mm} \pm 3,36$; facial de 121,06 $\mathrm{mm} \pm 9,18$; basal de $44,64 \mathrm{~mm} \pm 4,17$ e do neurocrânio de $64,48 \mathrm{~mm} \pm 4,69$. Os índices calculados também aparecem na tabela 1 .

Os resultados das medidas realizadas na área occipital são mostrados na tabela 2.

\section{Discussão}

Os crânios diferem no tamanho e na forma, muito mais nos cães domésticos do que em outras espécies de mamíferos ${ }^{1,16}$. A forma do crânio em cães mostra consideráveis variações raciais e individuais na forma e no tamanho ${ }^{11}$. A forma e a morfometria dos crânios de cães são parâmetros importantes para se determinar os diferentes tipos morfológicos. Na verdade, estes são os 
Tabela 1 - Medidas craniométricas em cães mesaticéfalos sem raça definida $(\mathrm{mm})$ - Botucatu - 2012

\begin{tabular}{lcc}
\hline & Média & desvio-padrão \\
\hline Comprimento do crânio & 177,72 & 16,22 \\
Comprimento neurocrânio & 96,84 & 7,25 \\
Comprimento viscerocrânio & 79,24 & 8,72 \\
Largura do neurocrânio & 62,24 & 3,8 \\
Largura zigomática & 95,44 & 7,85 \\
Altura do crânio & 41,92 & 3,9 \\
Comprimento da mandíbula & 126,79 & 16,3 \\
Comprimento da base do crânio & 140,24 & 12,21 \\
Comprimento condilobasal & 149,32 & 12,21 \\
Comprimento do focinho & 69,84 & 7,07 \\
Comprimento dos nasais & 50,08 & 6,77 \\
Comprimento do palato & 76,84 & 7,01 \\
Índice cranial & 53,83 & 3,36 \\
Índice neurocrânio & 64,48 & 4,69 \\
Índice facial & 121,06 & 9,18 \\
Índice basal & 44,64 & 4,17 \\
\hline
\end{tabular}

Tabela 2 - Área do forame magno em cães mesaticéfalos sem raça definida (mm) - Botucatu - 2012

\begin{tabular}{lcc}
\hline & Média & desvio-padrão \\
\hline Largura entre os processos jugulares & 41,00 & 4,2 \\
Largura entre os côndilos occipitais & 35,48 & 3,21 \\
Largura mastóide & 51,52 & 5,22 \\
Altura do triangulo occipital & 38,28 & 3,35 \\
Largura do forame magno & 17,48 & 2,27 \\
Altura do forame magno & 14,36 & 1,07 \\
Comprimento da chanfradura dorsal & 16,1 & 1,12 \\
Índice do forame magno & 83,71 & 14,44 \\
\hline
\end{tabular}

critérios mais importantes para se determinar o padrão racial dos cães e, os índices e as proporções raciais são usados para se determinar os tipos morfológicos ${ }^{1}$.

As várias mensurações craniométricas realizadas neste estudo, assim como os diversos índices craniais calculados foram obtidos com o objetivo de contribuir com uma possível padronização cranial de cães mesaticéfalos com ou sem raça definida. Todos os valores foram comparados com dados da literatura. Embora a denominação das mensurações, dos índices e/ ou das proporções possam diferir do apresentado por alguns relatos, os pontos utilizados e medidos correspondem aos usuais apresentados pela literatura.

Os índices obtidos nos crânios mesaticéfalos são comparados com os índices obtidos em crânios de cães de raças definidas e calculados por outros autores. Estas comparações são apresentadas na tabela 3. $\mathrm{O}$ índice cranial médio em crânios mesaticéfalos de cães sem raça definida foi de $53,83 \mathrm{~mm}$, ao passo que, Evans ${ }^{1}$, cita o índice cranial médio para o tipo de crâ- 


\begin{tabular}{|c|c|c|}
\hline Referências & Raças mesaticéfalas & Média $(\mathrm{mm})$ \\
\hline Cães mesaticéfalos & sem raça definida & $53,83 \pm 3,36$ \\
\hline \multirow[t]{2}{*}{ Stockard (1941) } & German Sheepdog & 51,33 \\
\hline & Great Dane & 51,67 \\
\hline \multirow[t]{3}{*}{ Komeyli (1984) } & Dachshund & 54,64 \\
\hline & Dalmáta & 56,39 \\
\hline & German Sheepdog & 50,59 \\
\hline \multirow[t]{2}{*}{ Sisson (1986) } & Fox Terrier & 70 \\
\hline & Pomeraniano branco & $72-75$ \\
\hline Onar (1999) & German Shepherd dog & 51,44 \\
\hline \multirow[t]{2}{*}{ Evans (1993) } & German Shepherd, Beagle e Setter & 51 \\
\hline & Outras raças mesaticéfalas & 52 \\
\hline
\end{tabular}

nio mesaticéfalo como sendo $52 \mathrm{~mm}$. Além disso, o índice cranial encontrado nos cães sem raça definida, deste estudo, esteve próximo aos índices relatados em crânios de cães das raças German Shepherd, Beagle e Setter ${ }^{1,8}$, porém foi bem diferente do encontrado nas raças Fox Terrier e Pomeraniano branco, enquadradas também como mesaticéfalas ${ }^{1}$. Neste estudo, ao comparar os resultados encontrados, optou-se por considerar as raças German Shepherd, Beagle e Setter como exemplos de raças com crânios mesaticéfalos ${ }^{1,6}$, embora outros autores possam considerar o German Shepherd como pertencente ao grupo dolicocéfalo ${ }^{8,17}$.

Os índices do neurocrânio $(64,48 \mathrm{~mm} \pm 4,69)$, facial $(121,06 \mathrm{~mm} \pm 9,18)$ e basal $(44,64 \mathrm{~mm} \pm 4,17)$ dos crânios mesaticéfalos em cães sem raça definida, obtidos neste estudo, estão próximos aos limites considerados para crânios de raças mesaticéfalas, que são, em média, $56 \mathrm{~mm}$ para o índice do neurocrânio, $111 \mathrm{~mm}$ para o facial, e $52 \mathrm{~mm}$ para o basal ${ }^{1}$. Não havendo, portanto, diferença significativa entre eles.

Nos 25 crânios mesaticéfalos estudados, observou-se a chanfradura ou incisura dorsal. No entanto,
Janeczek et al. ${ }^{15}$, ao estudar o forame magno em 37 crânios de cães da idade do ferro, encontrados na Turquia, observou a incisura dorsal em apenas sete cães. Estes autores não citam o tipo de crânio usado na pesquisa. Simoens, Poels e Pazvant ${ }^{10}$ e Watson, Lahunta e Evans ${ }^{18}$ observaram a incisura dorsal em Beagles e Pequinês, embora com certa variabilidade. Estes autores afirmaram que a incisura dorsal é uma variação anatômica da área do forame magno em cães. Há relatos que sugerem que a incisura dorsal está ausente ou é rara, quando presente, em raças mesaticéfalas e dolicocéfalas, e pode ser uma característica específica da raça resultante da domesticação ${ }^{15}$. Segundo Evans ${ }^{1}$, raças mesaticéfalas de cães apresentam a incisura dorsal diferentemente de outras raças, onde o forame magno é circular. Neste estudo, observou-se forame magno com forma ovalada e com incisura dorsal característica, ligeiramente circular.

Embora com algumas diferenças nas mensurações, não houve diferença significativa entre as dimensões observadas e as relatadas para crânios mesaticéfalos. 


\section{Referências}

1. EVANS, H. E. Miller's anatomy of the dog. 3. ed. Philadelphia: Saunders Company, 1993. p. 128-163.

2. CONSTANTINESCU, G. M. Anatomia clínica de pequenos animais. Rio de Janeiro: Guanabara-Koogan, 2005. p. 130-132.

3. SILVA MACHADO, T. F. Estudo comparativo da localização do seio venoso sagital dorsal no crânio de cães braquiocefálicos e mesaticefálicos para craniotomia transfrontal. 2006. 102 p. Dissertação (Mestrado) - Faculdade de Medicina Veterinária e Zootecnia, Universidade de São Paulo, São Paulo, 2006.

4. HOFMANN-APPOLLO, F. Estudo comparativo da forma do crânio de cães braquiocefálicos e mesaticefálicos por meio de técnica de morfometria geométrica em três dimensões. 2009. 101 p. Dissertação (Mestrado) - Faculdade de Medicina Veterinária e Zootecnia, Universidade de São Paulo, São Paulo, 2009.

5. STOCKARD, C. R. The genetic and endocrine basis for differences in form and behavior. Philadelphia: Wistar Institute, 1941. p. 1-775.

6. BREHM H.; LOEFFLER, K.; KOMEYLI, H. Skull shape in the dog. Anatomia, Histologia, Embryologia, v. 14, p. 324-331, 1985.

7. REGEDON, S.; ROBINA, A.; FRANCO, A.; VIVO, J. M.; LIGNEREUX, Y. Détermination radiologique et statistique dês types morphologiques crâniens chez le chien: dolichocéphalie, mésocéphalie et brachycéphalie. Anatomia, Histologia, Embryologia, v. 20, n. 2, p. 129-138, 1991.

8. ONAR, V. A morphometric study on the skull of the german shepherd dog (Alsatian). Anatomia, Histologia, Embryologia, v. 28, n. 4, p. 253-256, 1999.

9. ONAR, V.; ÖZCAN, S.; PAZVANT, G. Skull typology of adult male kangal dogs. Anatomia, Histologia, Embryologia, v. 30, n. 1, p. 41-48, 2001.
10.SIMOENS, P.; POELS, P.; LAUWERS, H. Morphometric analysis of the foramen magnum in Pekingese dogs. American Journal of Veterinary Research, v. 55, n. 1, p. 34-39, 1994.

11.SISSON, S. Osteologia do carnívoro. In: SISSON, S.; GROSSMAN, J. D. Anatomia dos animais domésticos, 5. ed. Rio de Janeiro: Guanabara-Koogan, 1986. v. 2, p. 1377-1391.

12.NICKEL, R.; SCHUMMER, A.; WILLE, H. Passiver bewegungsapparat, skelettsystem. In: NICKEL, R.; SCHUMMER, A.; SEIFERLE, E. Lehrbuch der anatomie der haustiere. Berlin: Verlag Paul Parey, 1977. p. 150-151.

13.REGEDON, S.; FRANCO, A.; LIGNEREUX, Y.; GARIN, J.; MARTIN, A. Skull, in Pekingese dog. Tomodensitometry and sex-linked difference. Revue de Medecine Veterinaire, v. 143, p. $745-748,1992$.

14.ONAR, V.; MUTU, R.; KAHVECIOGLU, K. O. Morphometric analysis of the foramen magnum in German Shepherd dogs (Alsatians). Annals of Anatomy, v. 179, n. 6, p. 563-568, 1997.

15.JANECZEK, M.; CHRÓSZCZ, A.; ONAR, V.; PAZVANT, G.; POSPIESZNY, N. Morphological analysis of the foramen magnum of dogs from the iron age. Anatomia, Histologia, Embryologia, v. 37, n. 5, p. 359-361, 2008.

16. KOMEYLI, H. Nasennebenhölen bei dolicho-, meso und brachyzepahlen Hunden unter besonderer Berucksichtigung der rassesspezifischen Schädelformen. Giessen, Germany: Vet.-Med. University, 1984. p. 1-151.

17. ONAR, V.; GÜNES, H. On the variability of skull shape in German Shepherd (Alsatian) puppies. Anatomical Record, v. 272A, n. 1, p. 460-466, 2003.

18. WATSON, A. G. A.; LAHUNTA, A. de; EVANS, H. E. Dorsal notch of the foramen magnum due to incomplete ossification of supraoccipital bone in dogs. Journal of Small Animal Pratice, v. 30, n. 12, p. 666-673, 1989. 